\title{
3 Research Square

\section{Quantitative proteomics analysis of FFPE tumor samples reveals the influences of NET-1 siRNA nanoparticles and sonodynamic therapy on tetraspanin protein involved in HCC}

\section{Bolin Wu}

Harbin Mecical University Cancer Hospital

\section{Haitao Shang}

Harbin Medical University Cancer Hospital

Xitian Liang

Harbin Medical University Cancer Hospital

Huajing Yang Huajing Yang

Harbin Medical University Cancer Hospital

Hui Jing

Harbin Medical Universitu Cancer Hospital

Bo Li

Harbin Medical University Cancer Hospital

Wen Cheng ( $\nabla$ chengwen@hrbmu.edu.cn)

Harbin Medical University Cancer Hospital https://orcid.org/0000-0003-4294-9698

Research article

Keywords: hepatocellular carcinoma, tetraspanin protein, mass spectrometry, proteomics, ultrasound

Posted Date: October 1st, 2020

DOI: https://doi.org/10.21203/rs.3.rs-20923/v3

License: (c) (1) This work is licensed under a Creative Commons Attribution 4.0 International License.

Read Full License

Version of Record: A version of this preprint was published at Frontiers in Molecular Biosciences on May 10th, 2021. See the published version at https://doi.org/10.3389/fmolb.2021.678444. 


\section{Abstract}

Hepatocellular carcinoma (HCC) poses a severe threat to human health. The NET-1 protein has been proved to be strongly associated with HCC proliferation and metastasis in our previous study. Here, we established and validated NET-1 siRNA nanoparticles system to conduct targeted gene therapy of HCC xenograft in vivo with the aid of sonodynamic therapy (SDT). Then, a label-free proteome mass spectrometry workflow to analyze formalin-fixed and paraffin-embedded HCC xenograft samples collected in this study. The result showed that 78 proteins were differentially expressed after NET-1 protein inhibited. Among them, the expression of 61 proteins up-regulated and the expression of 17 proteins were significantly down-regulated. Of the differentially expressed proteins, the vast majority of Gene Ontology enrichment terms belong to the biological process. The KEGG pathway enrichment analysis showed that the 78 differentially expressed proteins significantly enriched in 45 pathways. We concluded that the function of the NET-1 gene is not only to regulate HCC but also to participate in a variety of biochemical metabolic pathways in the human body. Furthermore, the protein-protein interaction analysis indicated that the interactions of differentially expressed proteins are incredibly sophisticated. All the protein-protein interactions happened after the NET-1 gene has been silenced. Finally, our study also provides a useful proposal for targeted therapy based on tetraspanin proteins to treat $\mathrm{HCC}$, and further mechanism investigations are needed to reveal a more detailed mechanism of action for NET-1 protein regulation of HCC.

\section{Introduction}

Worldwide, liver cancer is the fourth most common cause of cancer-related death and ranks sixth in terms of incident cases(1). With a five-year survival of $18 \%$, liver cancer is the second most lethal tumor after pancreatic cancer. Hepatocellular carcinoma is a major type of primary liver cancer. Changes in protein expression accompany HCC progress; thus, some proteins can be used as potential biomarkers for diagnosis and treatment(2).

Sonodynamic therapy (SDT) was introduced by Yumita in 1989 (3). This recent non-invasive treatment is based on the photodynamic therapy (PDT) already well known in clinical practice, especially in the oncology field(4). SDT refers to a new anticancer strategy which uses non-thermal ultrasound energy in combination with drugs known as sonosensitizer agents (5-7). Normally, low-intensity focused ultrasound (LIFU) frequency is used for this technique $(1-3 \mathrm{MHz})$ to enhance the cavitation effect $(8,9)$. The sonosensitizer molecule together with US irradiation, under aerobic conditions, creates active oxygen species able to destroy the tumor tissues $(10,11)$. SDT exploits LIFU and sonosensitizer agents to cause profound physicochemical changes in cell structure.

Several reports have revealed that LIFU is able to enhance the anticancer effect of some chemotherapeutic drugs and improve cell membrane permeability. Our previous researches have proved that the combination of LIFU irradiation and nanobubble system is regarded as an efficient and safe 
method for gene transfection(12,13). Besides, LIFU combined NET-1 siRNA conjugated nanobubble system could effectively inhibit tumor growth and prolonged the life of experimental animals(14).

Neuroepithelial transforming gene 1 (NET-1) is located at chromosome 10p15 and encodes a 54-kDa oncoprotein(15). It is a guanine nucleotide exchange factor involved in cytoskeletal regulation and cancer cell invasion(16). All NET genes have initially been identified as EST clones with sequences homologous to tetraspan, a superfamily which is distinguished by the presence of four transmembrane domains and has been implicated in signal transduction, cell adhesion, migration, proliferation, and differentiation(17, 18). NET-1 protein was known to be a member of the tetraspanin family(19). NET-1 protein has been identified in HCC, where it is a mediator of invasion and metastasis(20-22). Our previous research proved that the NET-1 protein had an impactful role in the proliferation and stiffness of $\operatorname{HCC}(14,23)$. Besides, the low expression of NET-1 protein also reduced the migration and invasive ability of $\mathrm{HCC}(24)$. However, the potential carcinogenic mechanism of NET-1 protein is still unclear.

Proteomics is an efficient research tools to reveal the mechanism and pathogeny of diseases on the proteinic level. Because of proteomics analysis could analyze quite a lot of expressed proteins in tissues or cells, this revolutionary technology has been applied to identify HCC related proteins in many studies(25-27). However, altered expression of proteins quantified with conventional label-free proteomic methods was limited by the fresh or rapidly frozen tissue samples. Most human tumor samples archived in hospitals for pathologic diagnosis are Formalin-Fixed Paraffin-Embedded (FFPE), which have been globally used for DNA, RNA, protein, and morphological measurements, and preanalytical factors affecting each type of measure have been identified(28). Besides, multifarious enlightened techniques have been invented for genomic $(29,30)$, transcriptomic $(31)$, proteomic and protein $(32,33)$ from FFPE samples. For the first time, FFPE tissues have been analyzed for protein using antibodies in 1991 as the invention of the heat-induced antigen retrieval (HIAR) technique for immunohistochemistry (IHC)(34). Kinds of different technologies have been applied to extract proteins from FFPE samples, which have extended the research of proteins to a proteomic level.(35-37). These studies have initially confirmed that FFPE samples can be used in mass spectrometry-based proteomic analysis.

Here, we established and validated NET-1 siRNA nanoparticles system, which were then utilized for targeted gene delivery of HCC xenograft in vivo with the aid of SDT. Then, proteomic analyses of FFPE HCC xenograft samples were conducted to characterize the global quantitative protein expression profile and identify the differential protein expressions after gene therapy. Furthermore, we aimed to shed light on the functions of tetraspanin protein involved in HCC development, and in this way, reveal HCC-related proteins valuable for targeted therapy.

\section{Materials And Methods}

\section{Preparation of NET-1 siRNA nanoparticles}

The NET-1 siRNA duplex was designed according to our previous research(13). The nanoparticles were prepared with DSPG, DSPC, and DSPE-PEG2000, and the weight ratio was 7:2:1. All the phospholipids 
were purchased from Avanti Polar Lipids (Avanti Polar Lipids, Alabaster, AL, USA. 20 mg of phospholipids were dissolved in a mixed solution of chloroform and methyl alcohol. The mixed solution was subsequently purged by vacuum rotary evaporation to form phospholipid thin film. Then, the thin film was hydrated at $40^{\circ} \mathrm{C}$ with $5 \mathrm{~mL}$ of DEPC treated $\mathrm{H}_{2} \mathrm{O}$. This was followed by dissolving the appropriate amounts of NET-1 siRNA duplex in the lipid film solution. The NET-1 siRNA nanoparticles were obtained using an extrusion technology by mini-extruders (Avanti Polar Lipids, Alabaster, AL, USA) through a $400 \mathrm{~nm}$ membrane for 11 times. The obtained NET-1 siRNA nanoparticles were then transferred into a sealed vial and stored at $4^{\circ} \mathrm{C}$ for further experiments.

The structure of NET-1 siRNA nanoparticles was detected under transmission electron microscope (TEM, Hitachi TEM system, Japan). The size and zeta potential were investigated by dynamic light scattering (DLS) via the Malvern Zetasizer Nanoseries (Zeta PALS BI-90 Plus, Brookhaven Instruments).

\section{Cell lines and animal tumor inoculation}

The human HCC cell line HepG2 cells were a generous gift from the Institute of Cancer Research affiliated with Harbin Medical University (Harbin, China). Cells were cultured in DMEM medium supplemented with $10 \%$ fetal bovine serum and $1 \%$ penicillin/streptomycin in a humidified atmosphere containing $5 \%$ $\mathrm{CO}_{2} / 95 \%$ air at $37^{\circ} \mathrm{C}$.

BALB/c nude female mice (6-8week, 10-25 g) were purchased from Beijing Vital River Laboratory Animal Technology (Beijing, China). All the animals were housed in an environment with a temperature of $22 \pm 1^{\circ} \mathrm{C}$, a relative humidity of $50 \pm 1 \%$, and a light/dark cycle of $12: 12 \mathrm{~h}$. All animal studies (including the mice euthanasia procedure) were done in compliance with the regulations and guidelines of Harbin Medical University institutional animal care and conducted according to the Association for Assessment and Accreditation of Laboratory Animal Care and the Institutional Animal Care and Use Committee guidelines. Mice were anesthetized with $3 \%$ isoflurane inhalation and $1 \mathrm{~L} / \mathrm{min} 100 \%$ oxygen. $A$ total of $5 \times 10^{6}$ HepG2 cells were suspended in $50 \mathrm{ml}$ PBS and50 ml Matrigel (BD Biosciences, San Jose, CA, USA). Tumor cell-Matrigel mixture (100 ul) was subcutaneously injected in the right back position of the mice.

\section{In vivo studies}

Once the tumor diameter reached $0.5 \mathrm{~cm}$, the mice bearing tumors were randomly divided into 3 groups (8 mice/group): group A, blank (PBS); group B, NET-1 siRNA nanoparticles without LIFU irradiation; group C, NET-1 siRNA nanoparticles with LIFU irradiation. All the NET-1 siRNA nanoparticles were intravenously administered via the tail vein at a dose of $5 \mathrm{ml} / \mathrm{kg}$ body weight.

At 30 min postinjection, all groups were irradiated with LIFU using a CGZZ Low-Frequency Ultrasound Treatment Instrument (Institute of Ultrasound Imaging, Second Affiliated Hospital of Chongqing Medical University, Chongqing, China). LIFU parameters were as follows: frequency of $1 \mathrm{MHz}$, pulse repetition frequency of $1 \mathrm{kHz}$, duty cycle yield of $50 \%$, intensity of $1.0 \mathrm{~W} / \mathrm{cm}^{2}$, and duration of irradiation of $5 \mathrm{~min}$ per mouse. The nude mice were treated twice a week for a total of $60 \mathrm{~d}$. The survival end point was a 
tumor diameter of $20 \mathrm{~mm}$ in any direction (according to the Guidelines for Tumor Induction in Mice and Rats, American Association for Laboratory Animal Science, Memphis, TN, USA); the maximum tumor diameter was measured twice every week using Aixplorer US system with high-frequency probe (Super Linear TM SL15-4, Super Sonic Imagine, Aix-en-Provence, France). The HCC xenograft samples were harvested after euthanasia and formalin-fixed for long-stem storage and proteomic analysis.

\section{Proteomic Analysis}

\section{Total Protein Extraction}

Inspired by the survival result, FFPE HCC xenograft samples in group A and group C were selected for proteomic analysis. Three pieces of the FFPE samples in each groups were dewaxed with octane and then hydrated with graded ethanol. After hydration, the sample was washed twice with PBS. After removing the PBS solution, an appropriate amount of protein lysate ( $\%$ SDS, $100 \mathrm{mM}$ Tris, $\mathrm{pH}=8.5$ ) was added and incubated at $95^{\circ} \mathrm{C}$ for 10 minutes at room temperature, mixed by shaking, and sonicated in an ice-water bath for 5 minutes. The samples were de-crosslinked with a refractive index at $95^{\circ} \mathrm{C}$ for 60 min, then reduced by adding an appropriate amount of TCEP and carboxyamidomethylated in CAA at $95^{\circ} \mathrm{C}$ for $5 \mathrm{~min}$. The samples were sequentially centrifuged at $12000 \mathrm{~g}$ at $4^{\circ} \mathrm{C}$ for $15 \mathrm{~min}$. Collecting the supernatant and adding four times volume of pre-cooling acetone at $-20^{\circ} \mathrm{C}$, and precipitated it at $-20^{\circ} \mathrm{C}$ for at least $4 \mathrm{~h}$. Centrifuging at $12000 \mathrm{~g}$ for $15 \mathrm{~min}$ at $4^{\circ} \mathrm{C}$. Collect the precipitate and air drying. An appropriate amount of protein solution ( $6 \mathrm{M}$ urea, $100 \mathrm{mM} \mathrm{TEAB}, \mathrm{pH}=8.5$ ) was added to dissolve the protein pellet.

\section{Trypsin treatment}

The protein solution was added to flat membrane ultrafiltration (cut off molecular is $10 \mathrm{kDa}$ ) tube and was centrifuged at $14,000 \mathrm{~g}$ at room temperature for 20 minutes, and the flow-through was discarded. $100 \mu \mathrm{L}$ of $50 \mathrm{mM}$ TEAB was added, and the sample was centrifuged at $14000 \mathrm{~g}$ at room temperature for 20 minutes. The washing procedure was repeated four times. $100 \mu \mathrm{L}$ of $50 \mathrm{mM}$ TEAB and an amount of 1:50 mass ratio of trypsin were added to the protein and incubated at $37^{\circ} \mathrm{C}$ overnight. After being centrifuged at $14000 \mathrm{~g}$ for $20 \mathrm{~min}$, an equal volume of $2 \%$ formic acid was added. After mixing, the solution was centrifuged at $14,000 \mathrm{~g}$ for $20 \mathrm{~min}$ at room temperature. The supernatant of flow-through was slowly passed through a $\mathrm{C} 18$ desalting column, and then $1 \mathrm{~mL}$ washing solution $(0.1 \%$ formic acid, $4 \%$ acetonitrile) was added to wash three times in succession, then $0.4 \mathrm{~mL}$ of eluent $(0.1 \%$ formic acid, $75 \%$ acetonitrile) was added to elute twice in sequence, the eluent samples were combined and freezedried.

\section{LC-MS/MS Analysis}

Mobile phase $A$ ( $100 \%$ water, $0.1 \%$ formic acid) and B solution ( $80 \%$ acetonitrile, $0.1 \%$ formic acid) were prepared. The lyophilized powder was dissolved in $10 \mu \mathrm{L}$ of solution $\mathrm{A}$, centrifuged at $15,000 \mathrm{rpm}$ for 20 min at four ${ }^{\circ} \mathrm{C}$, and $1 \mu \mathrm{g}$ of the supernatant was injected into a home-made C18 Nano-Trap column (2 
$\mathrm{cm} \times 75 \mu \mathrm{m}$, three $\mu \mathrm{m})$. Peptides were separated in a home-made analyticalcolumn $(15 \mathrm{~cm} \times 150 \mu \mathrm{m}, 1.9$ $\mu \mathrm{m})$ using linear gradient elution, as listed in Supplemental Table 1. The isolated peptides were analyzed by the Q Exactive series mass spectrometer (Thermo Fisher), with ion source of Nanospray Flex ${ }^{\mathrm{TM}} \mathrm{QESI} \mathbb{\mathrm { Q }}$, spray voltage of $2.3 \mathrm{kV}$, and ion transport capillary temperature of $320^{\circ} \mathrm{C}$. Full scan range from $\mathrm{m} / \mathrm{z} 350$ to 1500 with resolution of 60000 (at $\mathrm{m} / \mathrm{z} 200$ ), an automatic gain control (AGC) target value was $3 \times 106$, and a maximum ion injection time was $20 \mathrm{~ms}$. The top 20ه40》 precursors of the highest abundant in the full scan were selected and fragmented by higher-energy collisional dissociation (HCD) and analyzed in MS/MS, where resolution was 15000 (at m/z 200), the automatic gain control (AGC) target value was $5 \times 10^{4}$, the maximum ion injection time was $45 \mathrm{~ms}$, a normalized collision energy was set as $27 \%$, and intensity threshold was $2.2 \times 10^{4}$. The dynamic exclusion parameter was $20 \mathrm{~s}$. The raw data of M.S. detection was named as ".raw".

\section{Data analysis}

\section{The identification and quantitation of protein}

The resulting spectra from each fraction were searched separately against the homo sapiens uniprot database by the search engines: Proteome Discoverer 2.2 (PD 2.2, Thermo). The search parameters are set as follows: mass tolerance for precursor ion was ten $\mathrm{ppm}$, and mass tolerance for production was 0.02 Da. Carbamidomethyl was specified in PD 2.2 as fixed modifications. Oxidation of methionine (M) and acetylation of the $\mathrm{N}$-terminus was specified in PD 2.2 as variable modifications. A maximum of 2 missed cleavage sites was allowed.

The identified protein contains at least one unique peptide with FDR no more than 1.0\%. Proteins containing similar peptides that could not be distinguished by MS/MS analysis were identified as the same protein group. Precursor ion was quantified by a label-free quantification method based on intensity. Mann-Whitney Test statistically analyzed the protein quantitation results for proteins whose quantitation significantly different between experimental and control groups were defined as differentially expressed proteins (DEP).

\section{The functional analysis of protein and DEP}

Gene Ontology (GO) and InterPro (IPR) analysis were conducted using the interproscan-5 program against the non-redundant protein database (including Pfam, PRINTS, ProDom, SMART, ProSiteProfiles, PANTHER)(38), the databases of Clusters of Orthologous Groups (COG) and Kyoto Encyclopedia of Genes and Genomes (KEGG) were used to analyze the protein family and pathway. The probable proteinprotein interactions (PPI) were predicted using the STRING-db server(39) (http://string.embl.de/). The enrichment pipeline was used for enrichment analysis of G.O., IPR, and KEGG(40).

\section{Statistical analysis}


All data are presented as mean \pm standard error of the mean (SEM). Statistical analysis was performed by unpaired, two-tailed Students t-test using the GraphPad Prism 8.0 software (GraphPad Software) if not denoted otherwise. Differences were considered statistically significant at $p$-value $<0.05$. Mann-Whitney Test statistically analyzed the protein quantitation results. Because $>2$ groups were compared in this survival study, the log-rank test was used to compare 2 specific groups when the overall values were $p<$ 0.05 . Proteins were supposed to be significantly differentially expressed when the $p$-value $<0.05$, Fold Change $(F C) \leq 0.05$ or $p$-value $<0.05, F C \geq 2.0$.

\section{Results}

\section{Characterization of NET-1 siRNA nanoparticles}

The obtained NET-1 siRNA nanoparticles were well dispersed in aqueous solution and appeared as quasispheres with nanosize by transmission electron microscopy observation (Fig. 1A). The mean particlesize of TNB was $675.1 \pm 33.3 \mathrm{~nm}$, with a 0.341 mean polydispersity index (Fig. 1B).

\section{Analysis of in vivo study}

A decrease in tumor growth was observed in group $C$ compared with other groups $(P=0.0384$, Fig. $2 A)$, whereas no noticeable difference was observed between group $A$ and group $B(P=0.6667)$.

During the 60-d follow-up, all mice with a tumor larger than $2 \mathrm{~cm}$ were euthanized (according to the Guidelines for Tumor Induction in Mice and Rats).

Briefly, of group A, 3 mice died a natural death and 3 mice were euthanized within $45 \mathrm{~d}$, the median survival was $28 \mathrm{~d}$ (Fig. 2B, black). Of group B, 4 mice died a natural death within $35 \mathrm{~d}$ (Fig. 2B, blue), and the other 2 mice were euthanized owing to the tumor size (Fig. 2A, blue).

Conversely, the group $C$ showed the best survival result. 4 mice in group $C$ survived until the end of followup (Fig. 2B, red), and none of the tumors on any of the mice exceeded $20 \mathrm{~mm}$ in any direction during the follow-up period (Fig. 2A, red). The log-rank (Mantel-Cox) test showed that the survival curves were significantly different $(P=0.0019)$.

\section{Analysis of differential protein expression}

In this study, we carried out a label-free proteome analysis using FFPE HCC xenograft samples. In total, 3389 proteins were quantified from the label-free analysis. Compared to Group A to Group B, a total of 78 proteins were differentially expressed ( $p$-value $\leq 0.05$, Fig $3 A$ ). Among them, the expression of 61 proteins, such as P04350, M0QZR4, and A0A024R056, were significantly up-regulated (FC $\geq 2.0$, $p$-value $\leq 0.05)$, and the manifestation of 17 proteins, such as A8K335, Q6NVC0 and A0A024QZY5, were significantly downregulated ( $F C \leq 0.05$, $p$-value $\leq 0.05)$. Cluster analysis of differentially expressed mRNAs and IncRNAs was shown by a heat map (Fig 3B). Red indicates high expression proteins, and blue indicates low expression proteins. 


\section{Analysis of G.O. Functional Enrichment}

Based on the G.O. enrichment analysis, we can explore the main biological functions of DEP. The representative five enriched G.O. details of DEP were shown in table 1. The top 20 G.O. enrichment terms of DEP were illustrated in Fig 4. The vast majority of G.O. enrichment terms belong to the biological process (B.P.). The enriched G.O. terms of the biological process were exhibited detailed in Directed Acyclic Graph (DAG), as shown in Supplemental Fig 1A. Besides, the enriched G.O. terms of molecular function (M.F.) and cellular component (CC) were shown in Supplemental Fig 1B, C, respectively. The complete result of G.O. enrichment analysis was shown in Supplemental Table 2. The top 10 G.O. enrichment terms were presented graphically by DAG (Supplemental Fig.1).

\section{Analysis of KEGG Pathway Enrichment}

The dominant biochemical metabolic pathways and signal transduction pathways, which were regulated by DEP, could be identified by KEGG pathway enrichment analysis. The representative five enriched KEGG pathways of DEP were "Glutamatergic synapse", "Endocrine resistance”, "GABAergic synapse”, "Gap junction" and "Melanogenesis" (table 2). The top 20 enriched KEGG pathway terms were presented by a scatter plot (Fig. 5). The details of all KEGG pathway enrichment analysis were shown in Supplemental Table 3.

\section{Analysis of IPR Enrichment}

In this study, we identified 47 IPR terms with differential enrichment (Supplemental Table 4). The representative five enriched IPR IDs, and titles of DEP were shown in Table 3. The top 20 enriched IPR terms were explained in Fig.6. The details of all IPR enrichment terms were explained in Supplemental Table 4.

\section{Protein-protein interaction analysis}

PPI analysis indicated that the significantly up-regulated proteins P12931, A0A2R8Y5A3, Q00534, and P27361 and the significant down-regulated proteins P31415, A0A024QZY5, B7Z9B1, B4DL07, and A8K335 were interrelated and interacted with each other (Fig. 7).

\section{Discussion}

In our previous study, the NET-1 protein functions of HCC were investigated in vivo(23). The expression of NET-1 protein in HCC xenograft was merely detected by IHC staining. In the present study, we hypothesized that the combination of NET-1 siRNA nanoparticles system and SDT could effectively improve transfection efficiency and regulate multiple proteins. Label-Free proteome analysis was conducted to identify the differentially expressed proteins. Surprisingly, a total of 78 DEP were sifted from 3389 quantified proteins ( $p$-value $\leq 0.05$ ). Moreover, 61 proteins were significantly up-regulated (red dots in Fig 3A, FC $\geq 2.0, p$-value $\leq 0.05$ ), and 17 proteins were significantly down-regulated (green dots in Fig $3 \mathrm{~A}$, $\mathrm{FC} \leq 0.05$, $p$-value $\leq 0.05$ ). Our hypothesis was preliminarily confirmed preliminarily. Encouraged by the 
proof-of-concept results, we analyzed the potential functions of DEP based on the mass spectra proteome analysis results.

Firstly, G.O. enrichment analysis for biological process, cell component, and molecular function was performed. A total of $78 \mathrm{G}$.0. enrichment terms belong to the biological process, which would indicate the most DEP was involved in regulating the biological process of HCC. On the other hand, NET-1 protein was a member of the tetraspanin family, which was is a crucial point of HCC biological processes such as proliferation, differentiation, migration, and invasion $(41,42)$. Consequently, silencing the NET-1 gene could regulate many HCC biological processes by depressing the NET-1 protein expression. Secondly, KEGG pathway enrichment analysis was performed to explore the pathways, which may be regulated by the NET-1 gene. A total of 45 significantly enriched pathways were identified ( $p$-value $\leq 0.05$ ), as shown in Supplemental Table 3. In organisms, different proteins co-ordinate their biological behaviors, and the pathway-based analysis helps to understand their biological functions further. Interestingly, the DEP enriched in multifarious pathways, not only cancer-related pathways but also many other pathways, such as "Olfactory transduction" (map04740), "Cocaine addiction" (map05030), "Morphine addiction" (map05032), "Kaposi's sarcoma-associated herpesvirus infection" (map05167) and "Human papillomavirus infection Human papillomavirus infection" (map05165). We concluded that the function of the NET-1 gene is not only to regulate HCC but also to participate in a variety of biochemical metabolic pathways in the human body. Thirdly, proteins are composed of IPR that are units of protein structure, function, and evolution. The study of the IPR of proteins is essential for understanding the biological role of proteins and their development. In this study, 47 IPR enrichment terms were found, as shown in Supplemental Table 4 ( $p$-value $\leq 0.05$ ). Each IPR had a specific protein corresponding to it. It could be proved that IPR can form new proteins by copying and combining. The combination distribution between different IPR does not conform to the random model but shows that some IPR has a powerful combination ability, some of which are rarely combined with other domains. Finally, PPI analysis revealed the direct and potential regulatory relationships between DEP. As shown in Fig.7, all DEP formed a big circle, and each node represented a DEP. The up-regulated proteins are represented by red nodes, and the down-regulated proteins are represented by blue nodes. The interactions of DEP are incredibly sophisticated. For instance, the P27361 protein could regulate the other 13 DEP; the P12931 protein was regulated by the other 11 DEP. All the protein-protein interactions happened after the NET-1 gene has been silenced.

In summary, for the first time, our present study provides valuable insight into the regulation of NET-1 siRNA nanoparticles system and SDT on other proteins in HCC on a proteomics level. It proved that the NET-1 protein, one of the tetraspanin proteins, participated in regulating many critical signaling pathways in HCC development. The potential antitumor mechanism is that the tetraspanin protein regulated other proteins involved in the biological processes of HCC cells to inhibit proliferation and invasion. Our results also provide a useful proposal for targeted therapy based on tetraspanin proteins to treat HCC, and further mechanism investigations are needed to reveal a more detailed mechanism of action for NET-1 protein regulation of HCC. 


\section{Statements}

\section{Acknowledgment}

We thank Prof. Weiwei An and Prof. Yue Yang (Institute of Cancer Prevention and Treatment, Heilongjiang Academy of Medical Science) for supplying the altruistic guidance on the proteomic analyses.

\section{Statement of Ethics}

All animal studies (including the mice euthanasia procedure) were done in compliance with the regulations and guidelines of Harbin Medical University institutional animal care and conducted according to the AAALAC and the IACUC guidelines.

\section{Availability of data and materials}

The datasets used and analyzed during the current study are available from the corresponding author on reasonable request.

\section{Disclosure Statement}

The authors have no conflicts of interest to declare.

\section{Funding Sources}

This research was supported by the Natural Science Foundation of China (grant number 81873900), Project of Health Commission of Heilongjiang Province (grant number 2019053), and HAI YAN Science Foundation of Harbin Medical University Cancer Hospital (grant number JJQN2019-09 and JJQN201921).

\section{Author Contributions}

Conceptualization, W.C. and B.W.; Methodology, B.W., and H.S.; Data Curation, X.L.; Formal Analysis, X.L., and H.J.; Resources, B.W.; Writing - Original Draft, Y.T.; Visualization, Z.L., and B.L.; Funding Acquisition, W.C., H.S., X.L., and B.W.; Supervision, W.C. and B.W.

\section{References}

1. Villanueva A. Hepatocellular Carcinoma. The New England journal of medicine. 2019;380(15):145062.

2. Ferrin G, Aguilar-Melero P, Rodriguez-Peralvarez M, Montero-Alvarez JL, de la Mata M. Biomarkers for hepatocellular carcinoma: diagnostic and therapeutic utility. Hepatic medicine : evidence and research. 2015;7:1-10.

3. Yumita N, Nishigaki R, Umemura K, Umemura S. Hematoporphyrin as a sensitizer of cell-damaging effect of ultrasound. Japanese journal of cancer research : Gann. 1989;80(3):219-22. 
4. Brown SB, Brown EA, Walker I. The present and future role of photodynamic therapy in cancer treatment. The Lancet Oncology. 2004;5(8):497-508.

5. Castano AP, Demidova TN, Hamblin MR. Mechanisms in photodynamic therapy: part onephotosensitizers, photochemistry and cellular localization. Photodiagnosis and photodynamic therapy. 2004;1 (4):279-93.

6. Rosenthal I, Sostaric JZ, Riesz P. Sonodynamic therapy--a review of the synergistic effects of drugs and ultrasound. Ultrasonics sonochemistry. 2004;11(6):349-63.

7. McHale AP, Callan JF, Nomikou N, Fowley C, Callan B. Sonodynamic Therapy: Concept, Mechanism and Application to Cancer Treatment. Advances in experimental medicine and biology. 2016;880:42950 .

8. Shibaguchi H, Tsuru H, Kuroki M, Kuroki M. Sonodynamic cancer therapy: a non-invasive and repeatable approach using low-intensity ultrasound with a sonosensitizer. Anticancer research. 2011;31(7):2425-9.

9. Tachibana K, Feril LB, Jr., Ikeda-Dantsuji Y. Sonodynamic therapy. Ultrasonics. 2008;48(4):253-9.

10. Yumita N, Okudaira K, Momose Y, Umemura S. Sonodynamically induced apoptosis and active oxygen generation by gallium-porphyrin complex, ATX-70. Cancer chemotherapy and pharmacology. 2010;66(6):1071-8.

11. Yumita N, Iwase $\mathrm{Y}$, Nishi K, Komatsu H, Takeda K, Onodera $\mathrm{K}$, et al. Involvement of reactive oxygen species in sonodynamically induced apoptosis using a novel porphyrin derivative. Theranostics. 2012;2(9):880-8.

12. Wu B, Yuan $Y$, Han X, Wang Q, Shang H, Liang X, et al. Structure of LINC00511-siRNA-conjugated nanobubbles and improvement of cisplatin sensitivity on triple negative breast cancer. FASEB journal : official publication of the Federation of American Societies for Experimental Biology. 2020.

13. Wu B, Shang $H$, Liang $X$, Sun $Y$, Jing $H$, Han $X$, et al. Preparation of novel targeting nanobubbles conjugated with small interfering RNA for concurrent molecular imaging and gene therapy in vivo. FASEB journal : official publication of the Federation of American Societies for Experimental Biology. 2019:fj201900716RR.

14. Shang $H$, Wu $B$, Liang $X$, Sun $Y$, Han $X$, Zhang $L$, et al. Evaluation of therapeutic effect of targeting nanobubbles conjugated with NET-1 siRNA by shear wave elastography: an in vivo study of hepatocellular carcinoma bearing mice model. Drug Deliv. 2019;26(1):944-51.

15. Chan AM, Takai S, Yamada K, Miki T. Isolation of a novel oncogene, NET1, from neuroepithelioma cells by expression cDNA cloning. Oncogene. 1996;12(6):1259-66.

16. Murray D, Horgan G, Macmathuna P, Doran P. NET1-mediated RhoA activation facilitates lysophosphatidic acid-induced cell migration and invasion in gastric cancer. $\mathrm{Br} \mathrm{J}$ Cancer. 2008;99(8):1322-9.

17. Serru V, Dessen P, Boucheix C, Rubinstein E. Sequence and expression of seven new tetraspans. Biochimica et biophysica acta. 2000;1478(1):159-63. 
18. Kanetaka K, Sakamoto M, Yamamoto Y, Yamasaki S, Lanza F, Kanematsu T, et al. Overexpression of tetraspanin CO-029 in hepatocellular carcinoma. Journal of hepatology. 2001;35(5):637-42.

19. Ye K, Wang Z, Zhang G, Liang S. Prognostic significance of neuroepithelial transforming protein 1 in hepatocellular carcinoma. J Invest Surg. 2010;23(3):163-9.

20. Chen L, Wang Z, Zhan X, Li DC, Zhu YY, Zhu J. Association of NET-1 gene expression with human hepatocellular carcinoma. International journal of surgical pathology. 2007;15(4):346-53.

21. Wu YY, Chen L, Wang GL, Zhang YX, Zhou JM, He S, et al. Inhibition of hepatocellular carcinoma growth and angiogenesis by dual silencing of NET-1 and VEGF. Journal of molecular histology. 2013;44(4):433-45.

22. Shen SQ, Li K, Zhu N, Nakao A. Expression and clinical significance of NET-1 and PCNA in hepatocellular carcinoma. Medical oncology (Northwood, London, England). 2008;25(3):341-5.

23. Wu B, Shang H, Liang X, Sun $Y$, Jing $H$, Han $X$, et al. Preparation of novel targeting nanobubbles conjugated with small interfering RNA for concurrent molecular imaging and gene therapy in vivo. FASEB journal : official publication of the Federation of American Societies for Experimental Biology. 2019;33(12):14129-36.

24. Wu B, Qiao Q, Han X, Jing H, Zhang H, Liang H, et al. Targeted nanobubbles in low-frequency ultrasound-mediated gene transfection and growth inhibition of hepatocellular carcinoma cells. Tumour biology : the journal of the International Society for Oncodevelopmental Biology and Medicine. 2016;37(9):12113-21.

25. Chaerkady R, Harsha HC, Nalli A, Gucek M, Vivekanandan P, Akhtar J, et al. A quantitative proteomic approach for identification of potential biomarkers in hepatocellular carcinoma. J Proteome Res. 2008;7(10):4289-98.

26. Yang X, Xiong Q, Wu Y, Li S, Ge F. Quantitative Proteomics Reveals the Regulatory Networks of Circular RNA CDR1as in Hepatocellular Carcinoma Cells. J Proteome Res. 2017;16(10):3891-902.

27. Gao Q, Zhu H, Dong L, Shi W, Chen R, Song Z, et al. Integrated Proteogenomic Characterization of HBV-Related Hepatocellular Carcinoma. Cell. 2019;179(2):561-77.e22.

28. Bass BP, Engel KB, Greytak SR, Moore HM. A review of preanalytical factors affecting molecular, protein, and morphological analysis of formalin-fixed, paraffin-embedded (FFPE) tissue: how well do you know your FFPE specimen? Archives of pathology \& laboratory medicine. 2014;138(11):1520-30.

29. Martelotto LG, Baslan T, Kendall J, Geyer FC, Burke KA, Spraggon L, et al. Whole-genome single-cell copy number profiling from formalin-fixed paraffin-embedded samples. Nature medicine. 2017;23(3):376-85.

30. Van Allen EM, Wagle N, Stojanov P, Perrin DL, Cibulskis K, Marlow S, et al. Whole-exome sequencing and clinical interpretation of formalin-fixed, paraffin-embedded tumor samples to guide precision cancer medicine. Nature medicine. 2014;20(6):682-8.

31. Li P, Conley A, Zhang H, Kim HL. Whole-Transcriptome profiling of formalin-fixed, paraffin-embedded renal cell carcinoma by RNA-seq. BMC genomics. 2014;15:1087. 
32. Mantsiou A, Makridakis M, Fasoulakis K, Katafigiotis I, Constantinides CA, Zoidakis J, et al. Proteomics Analysis of Formalin Fixed Paraffin Embedded Tissues in the Investigation of Prostate Cancer. J Proteome Res. 2019.

33. Iglesias-Gato D, Wikstrom P, Tyanova S, Lavallee C, Thysell E, Carlsson J, et al. The Proteome of Primary Prostate Cancer. European urology. 2016;69(5):942-52.

34. Shi SR, Key ME, Kalra KL. Antigen retrieval in formalin-fixed, paraffin-embedded tissues: an enhancement method for immunohistochemical staining based on microwave oven heating of tissue sections. The journal of histochemistry and cytochemistry : official journal of the Histochemistry Society. 1991;39(6):741-8.

35. Broeckx V, Boonen K, Pringels L, Sagaert X, Prenen H, Landuyt B, et al. Comparison of multiple protein extraction buffers for GeLC-MS/MS proteomic analysis of liver and colon formalin-fixed, paraffinembedded tissues. Molecular bioSystems. 2016;12(2):553-65.

36. Fowler CB, O'Leary TJ, Mason JT. Improving the Proteomic Analysis of Archival Tissue by Using Pressure-Assisted Protein Extraction: A Mechanistic Approach. Journal of proteomics \& bioinformatics. 2014;7(6):151-7.

37. Wakabayashi M, Yoshihara H, Masuda T, Tsukahara M, Sugiyama N, Ishihama Y. Phosphoproteome analysis of formalin-fixed and paraffin-embedded tissue sections mounted on microscope slides. J Proteome Res. 2014;13(2):915-24.

38. Jones P, Binns D, Chang HY, Fraser M, Li W, McAnulla C, et al. InterProScan 5: genome-scale protein function classification. Bioinformatics (Oxford, England). 2014;30(9):1236-40.

39. Franceschini A, Szklarczyk D, Frankild S, Kuhn M, Simonovic M, Roth A, et al. STRING v9.1: proteinprotein interaction networks, with increased coverage and integration. Nucleic Acids Res. 2013;41(Database issue):D808-15.

40. Huang da W, Sherman BT, Lempicki RA. Bioinformatics enrichment tools: paths toward the comprehensive functional analysis of large gene lists. Nucleic Acids Res. 2009;37(1):1-13.

41. Mazzocca A, Liotta F, Carloni V. Tetraspanin CD81-regulated cell motility plays a critical role in intrahepatic metastasis of hepatocellular carcinoma. Gastroenterology. 2008;135(1):244-56.e1.

42. Mazzocca A, Birgani MT, Sabba C, Carloni V. Tetraspanin-enriched microdomains and hepatocellular carcinoma progression. Cancer letters. 2014;351(1):23-9.

\section{Tables}

Table 1 the representative 5 enriched GO terms 


\begin{tabular}{|c|c|c|c|c|}
\hline GO ID & GO Term & $\begin{array}{l}\text { GO } \\
\text { Class }\end{array}$ & P value & Protein ID \\
\hline GO:0006468 & protein phosphorylation & $\mathrm{BP}$ & 0.001063999 & $\begin{array}{l}\text { Q00534, P27361, F8W6G1, A0A024QZY5, } \\
\text { O14976, B4E2LO, P12931 }\end{array}$ \\
\hline G0:0004672 & protein kinase activity & MF & 0.001220283 & $\begin{array}{l}\text { Q00534, P27361, F8W6G1, A0A024QZY5 } \\
\text { O14976, B4E2L0, P12931 }\end{array}$ \\
\hline $\begin{array}{l}\text { G0:0034329 } \\
\text { GO:0042803 }\end{array}$ & $\begin{array}{l}\text { cell junction assembly } \\
\text { protein } \\
\text { homodimerization } \\
\text { activity }\end{array}$ & $\begin{array}{l}\text { BP } \\
\text { MF }\end{array}$ & $\begin{array}{l}0.002098766 \\
0.003455028\end{array}$ & $\begin{array}{l}\text { A0A2R8Y5A3, P78357 } \\
\text { B7Z9B1, B4DL07 }\end{array}$ \\
\hline GO:0033270 & paranode region of axon & CC & 0.019097794 & P78357 \\
\hline
\end{tabular}

CC represents Cellular Component, MF represents Molecular Function, BP represents Biological Process.

Table 2 The representative 5 enriched KEGG pathway terms of DEP

\begin{tabular}{|c|c|c|c|c|}
\hline Map ID & Map Title & $P$ value & Protein ID & Description \\
\hline map04724 & $\begin{array}{l}\text { Glutamatergic } \\
\text { synapse }\end{array}$ & 0.000434861 & $\begin{array}{l}\text { A0A024R056, } \\
\text { P27361, } \\
\text { B4E2L0, } \\
\text { Q5U0L9 }\end{array}$ & $\begin{array}{l}\text { Guanine nucleotide binding protein (G protein), } \\
\text { beta polypeptide } 1 \text {, isoform CRA a, Mitogen- } \\
\text { activated protein kinase 3,cDNA FLJ54730, } \\
\text { highly similar to cAMP-dependent protein } \\
\text { kinase, beta-2-catalytic subunit, Homer } \\
\text { homolog } 3 \text { (Drosophila) }\end{array}$ \\
\hline map01522 & $\begin{array}{l}\text { Endocrine } \\
\text { resistance }\end{array}$ & 0.001179526 & $\begin{array}{l}\text { P27361, } \\
\text { P42773 } \\
\text { B4E2L0, } \\
\text { P12931 }\end{array}$ & $\begin{array}{l}\text { Mitogen-activated protein kinase 3,Cyclin- } \\
\text { dependent kinase } 4 \text { inhibitor C, cDNA FLJ54730, } \\
\text { highly similar to cAMP-dependent protein } \\
\text { kinase, beta-2-catalytic subunit, Proto-oncogene } \\
\text { tyrosine-protein kinase Src }\end{array}$ \\
\hline map04727 & $\begin{array}{l}\text { GABAergic } \\
\text { synapse }\end{array}$ & 0.001725647 & $\begin{array}{l}\text { A0A024R056 } \\
\text {,B4E2L0 } \\
\text { P12931, }\end{array}$ & $\begin{array}{l}\text { Guanine nucleotide binding protein (G protein), } \\
\text { beta polypeptide } 1 \text {, isoform CRA a, CDNA } \\
\text { FLJ54730, highly similar to CAMP-dependent } \\
\text { protein kinase, beta-2-catalytic subunit, Proto- } \\
\text { oncogene tyrosine-protein kinase Src }\end{array}$ \\
\hline map04540 & Gap junction & 0.001771527 & $\begin{array}{l}\text { P04350, } \\
\text { P27361 } \\
\text { B4E2L0, } \\
\text { P12931 }\end{array}$ & $\begin{array}{l}\text { Tubulin beta-4A chain, Mitogen-activated protein } \\
\text { kinase } 3 \text {,cDNA FLJ54730, highly similar to } \\
\text { cAMP-dependent protein kinase, beta-2-catalytic } \\
\text { subunit, Proto-oncogene tyrosine-protein kinase } \\
\text { Src }\end{array}$ \\
\hline map04916 & Melanogenesis & 0.003105093 & $\begin{array}{l}\text { A0A2R8Y5A3, } \\
\text { P27361 } \\
\text { B4E2L0 }\end{array}$ & $\begin{array}{l}\text { Catenin beta-1,Mitogen-activated protein kinase } \\
\text { 3,cDNA FLJ54730, highly similar to cAMP- } \\
\text { dependent protein kinase, beta-2-catalytic } \\
\text { subunit }\end{array}$ \\
\hline
\end{tabular}

Table 3 The representative 5 enriched IPR terms of DEP 


\begin{tabular}{|c|c|c|c|c|}
\hline IPR ID & IPR Title & P value & Protein ID & Description \\
\hline IPR001393 & Calsequestrin & 0.012354152 & P31415 & Calsequestrin-1 \\
\hline IPR002495 & Glycosyl transferase, family 8 & 0.012354152 & B2R5R5 & $\begin{array}{l}\text { cDNA, FLJ92583, highly } \\
\text { similar to Homo sapiens } \\
\text { qlycogenin (GYG), mRNA }\end{array}$ \\
\hline IPR003109 & GoLoco motif & 0.012354152 & AOAOAOMRC4 & $\begin{array}{l}\text { G-protein-signaling } \\
\text { modulator } 1\end{array}$ \\
\hline IPR003585 & $\begin{array}{l}\text { Neurexin/syndecan/glycophorin } \\
\text { C }\end{array}$ & 0.012354152 & P78357 & $\begin{array}{l}\text { Contactin-associated } \\
\text { protein } 1\end{array}$ \\
\hline IPR004134 & $\begin{array}{l}\text { Peptidase C1B, bleomycin } \\
\text { hydrolase }\end{array}$ & 0.012354152 & Q13867 & Bleomycin hydrolase \\
\hline
\end{tabular}

\section{Figures}




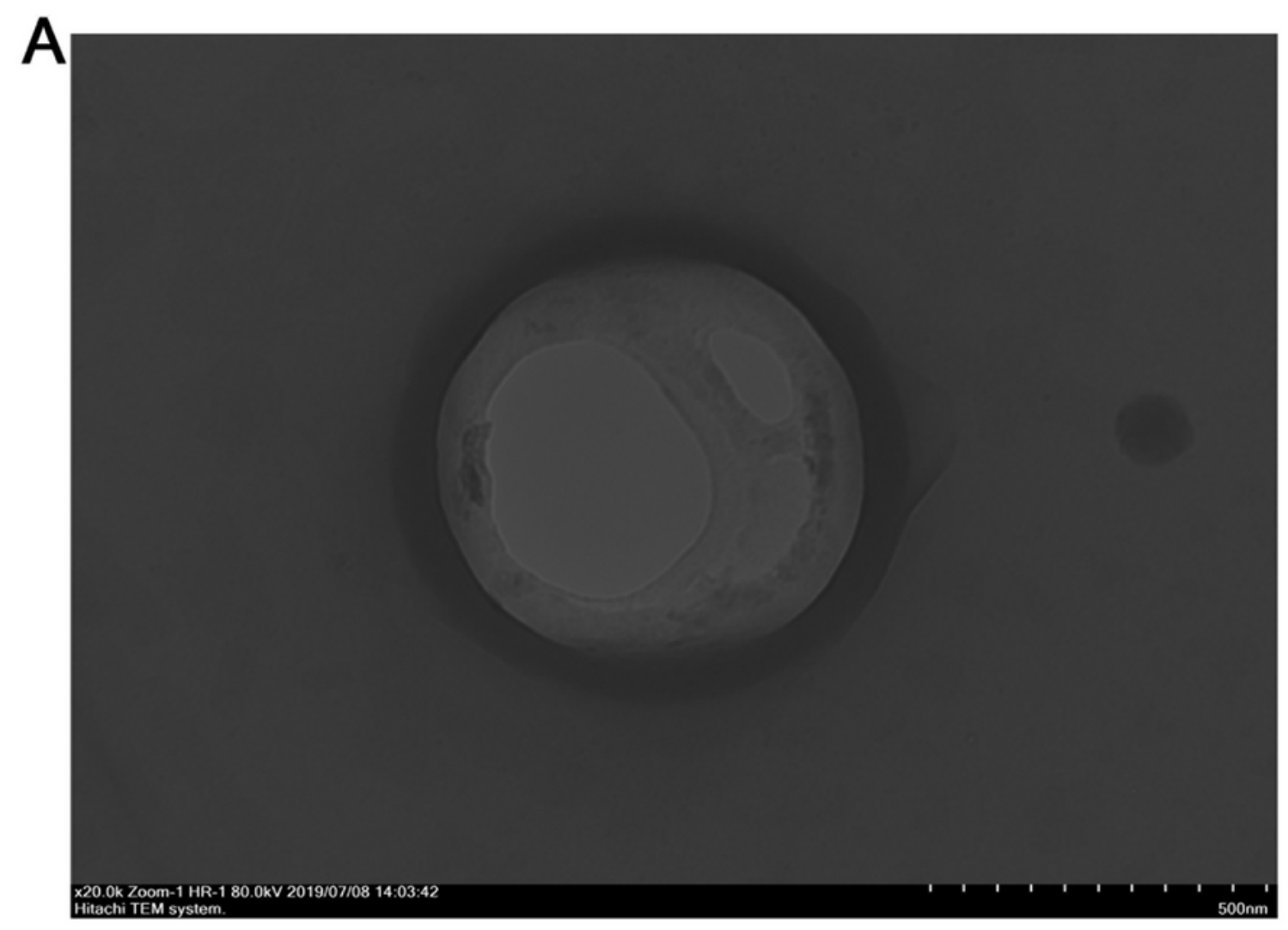

B

Effective Diameter: $675.1 \mathrm{~nm}$ Polydispersity: $\quad 0.341$

Avg. Count Rate: $\quad 57.0$ kcps

Baseline Index: $\quad 0.0$

Elapsed Time:

00:03:00

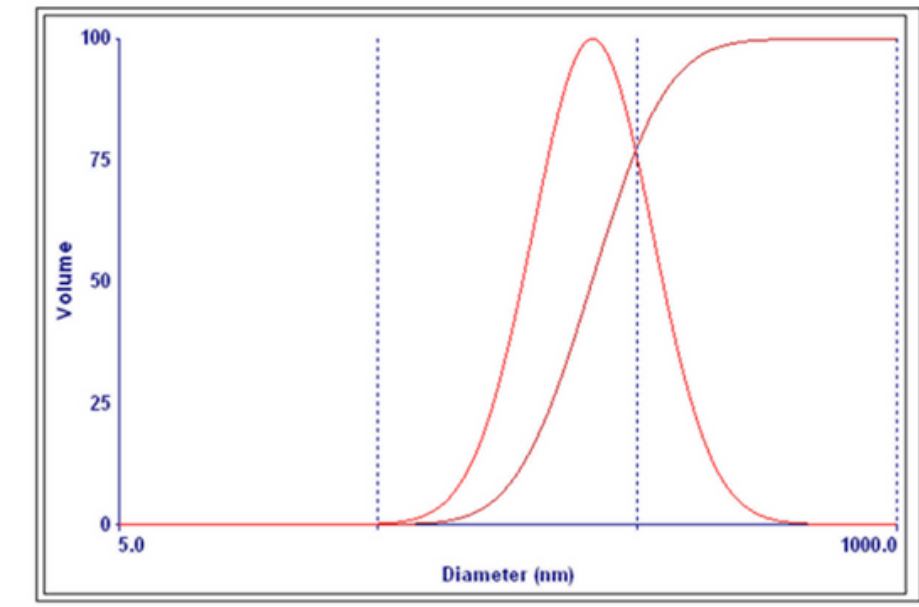

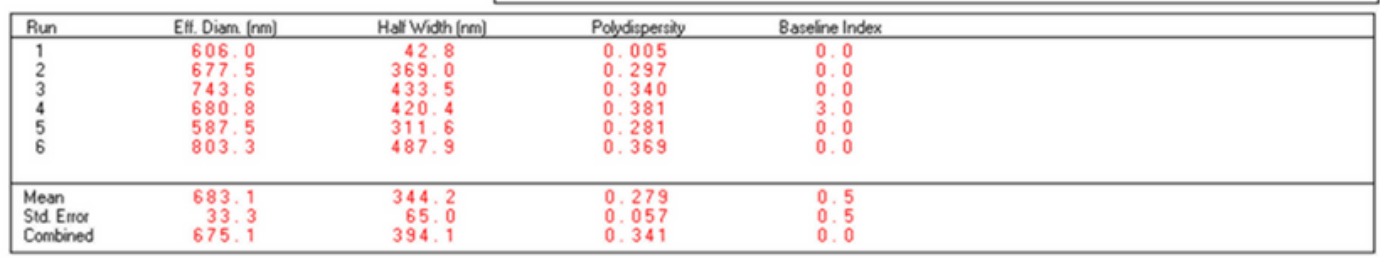

\section{Figure 1}

Structure and characterization of NET-1 siRNA nanoparticles. (A) Transmission electron microscopy image showed a quasi-spherical morphology of NET-1 siRNA nanoparticles with a diameter of about 600 $\mathrm{nm}$. Original magnification, 20,000X. Scale bar, $500 \mathrm{~nm}$; (B) The DLS results showed a mean particle size of NET-1 siRNA nanoparticles to be $675.1 \pm 33.3 \mathrm{~nm}$ with a 0.341 polydispersity value 


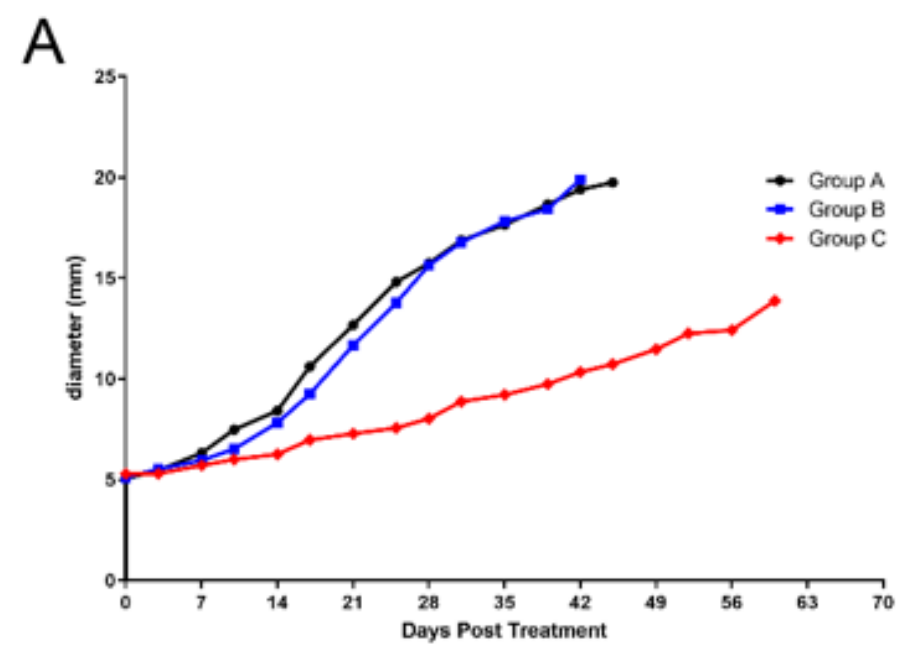

\section{B}

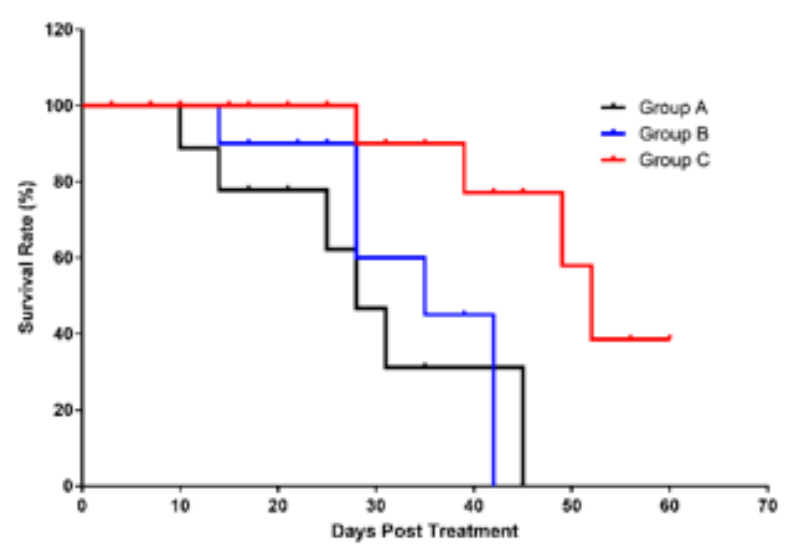

\section{Figure 2}

Tumor growth and mice survival in different mice groups. (A) Effect of the various treatments on tumor growth. (B) Effect of the various treatments on follow-up survival of mice ( $n=6 /$ group).
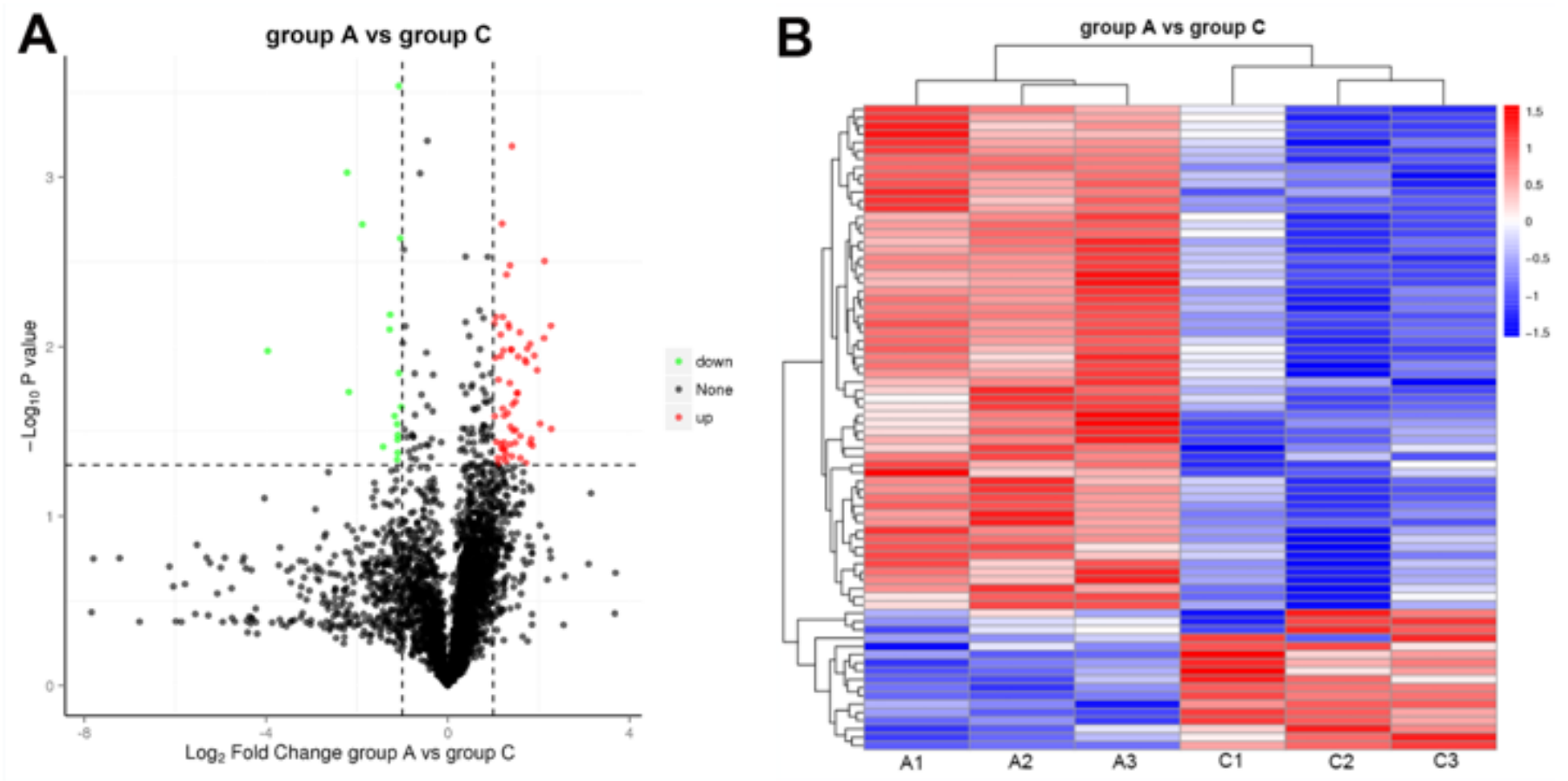

Figure 3

The illustration of DEP quantified from the label-free analysis. (A) Volcano plots of differentially expressed proteins between group 1 and group 2. Green dots represent down-regulated protein $(\mathrm{FC} \leq 0.05$, $p$-value $\leq 0.05)$, and red dots represent up-regulated protein ( $F C \geq 2.0, p$-value $\leq 0.05)$. (B) Heat map of hierarchical clustering of DEP. Red indicates high expression protein, and blue indicates low expression protein. 


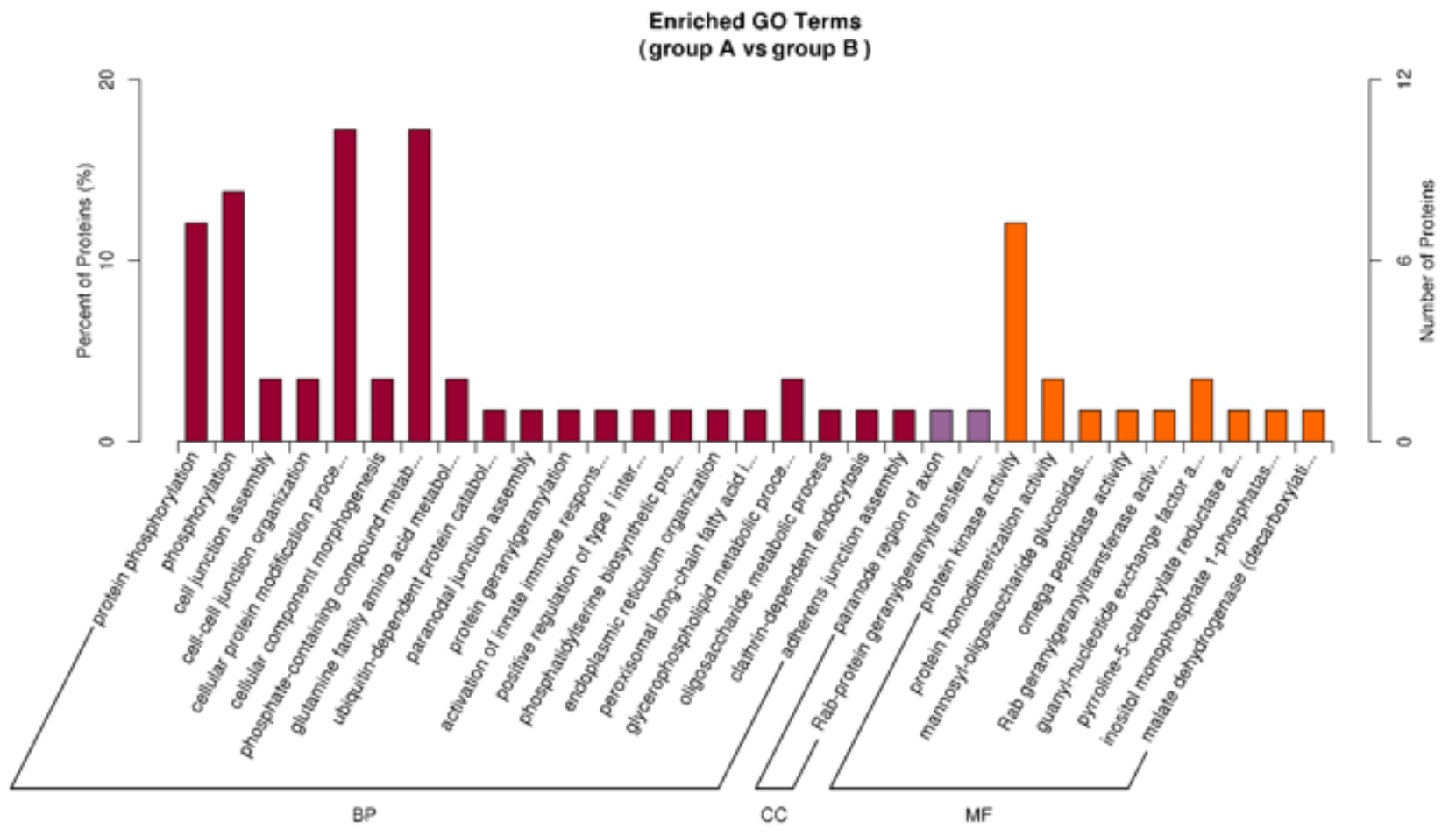

Figure 4

The illustration of the top 20 G.O. enrichment terms of all DEP. There are three categories of G.O. enrichment terms, biological process (B.P.), molecular function (M.F.), and cellular component (CC). The most DEP enriched in biological process (dark red), and only two CC terms had DEP enriched (purple). 


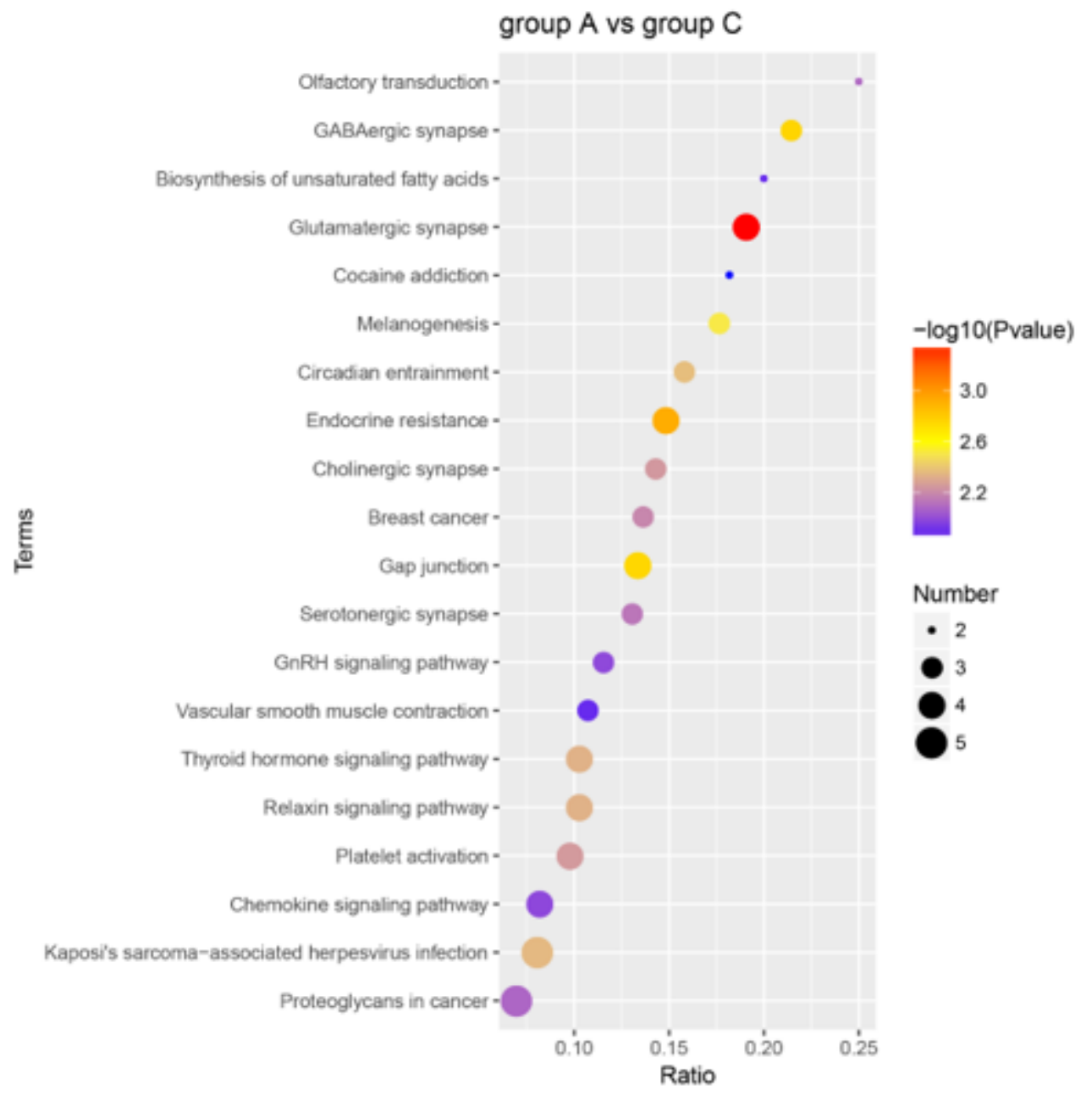

Figure 5

The KEGG pathway enrichment analysis of DEP. The top 20 KEGG enrichment terms of all DEP were shown in the scatter plot. The color of the dot represents the - log10(p-value), and the size of the dot represents the number of DEP. 


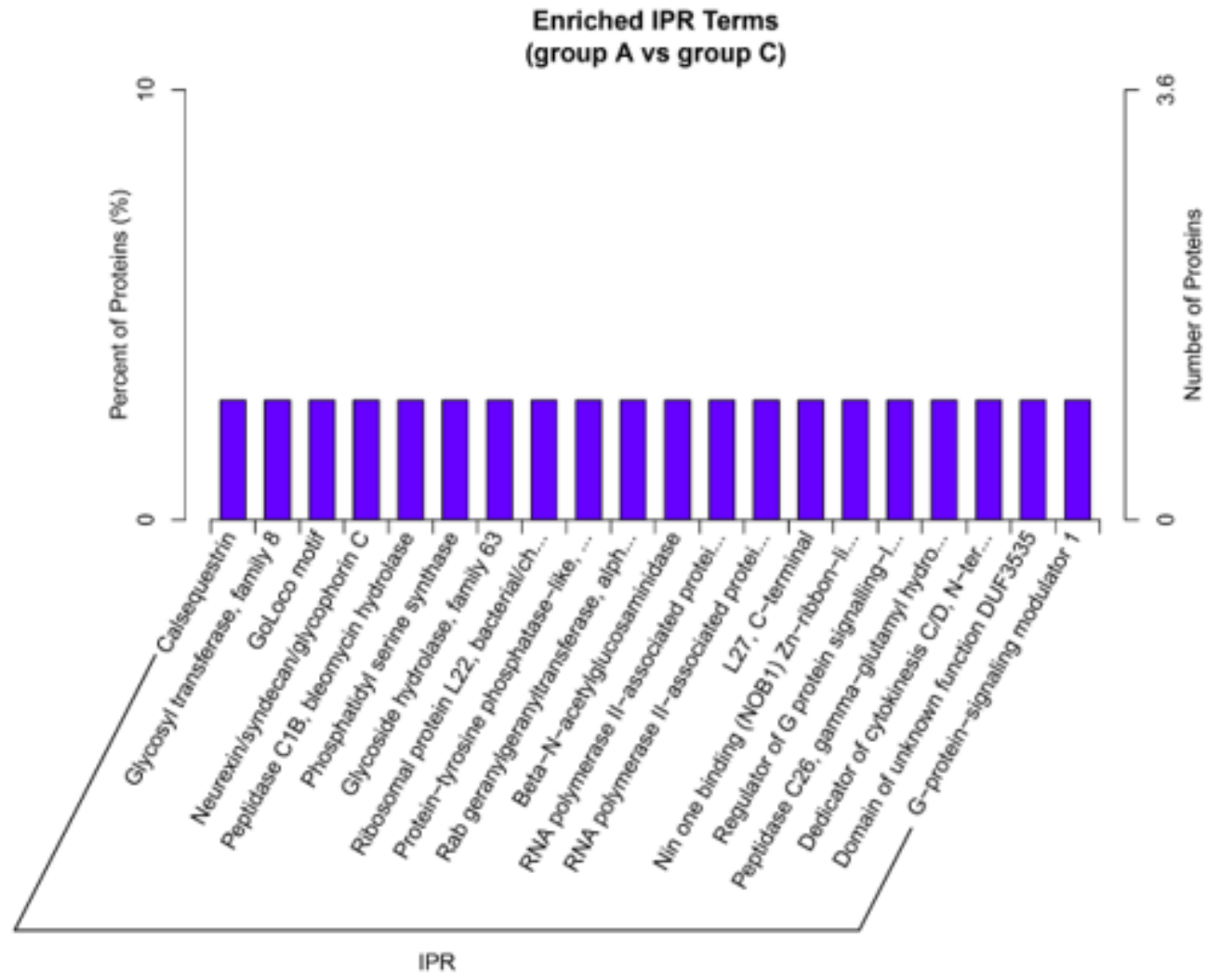

Figure 6

The IPR enrichment analysis. The top 20 IPR enrichment terms of all DEP were shown in the bar plot. Each IPR has only one DEP enriched.

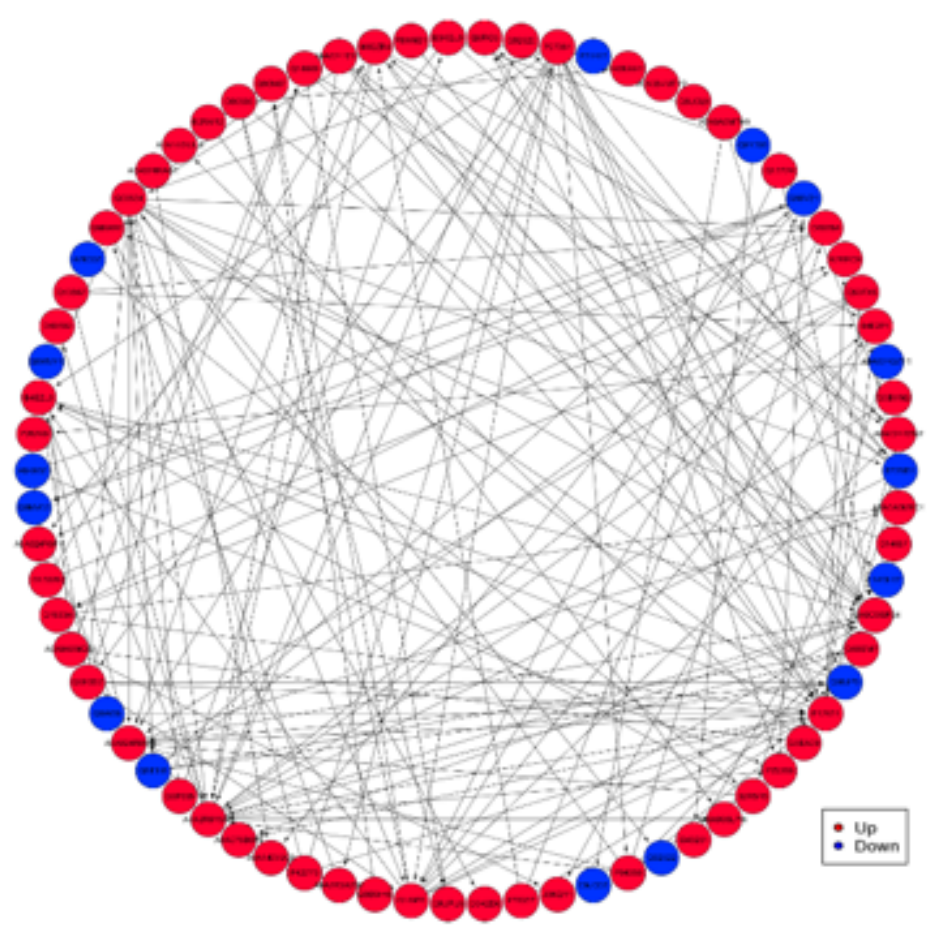

Figure 7 
An interaction network of PPI analysis. The network revealed the direct and potential regulatory relationships between DEP. The up-regulated proteins are represented by red nodes. The down-regulated proteins are represented by blue nodes

\section{Supplementary Files}

This is a list of supplementary files associated with this preprint. Click to download.

- Supplementalfigure1.tif

- SupplementalFigureCaptions.docx

- SupplementalTable1.docx

- SupplementalTable2.xlsx

- SupplementalTable3.xls

- SupplementalTable4.xls 